\title{
Erratum: Surface acoustic wave driven ferromagnetic resonance in nickel thin films: Theory and experiment [Phys. Rev. B 86, 134415 (2012)]
}

L. Dreher, M. Weiler, M. Pernpeintner, H. Huebl, R. Gross, M. S. Brandt, and S. T. B. Goennenwein (Received 17 July 2018; published 10 September 2018)

DOI: 10.1103/PhysRevB.98.099901

There are errors in some equations of this paper. The paper text and conclusions remain valid, but the following corrections should be made:

Equation (18) should read [1,2]

$$
\sigma_{i k}=\frac{1+\delta_{i k}}{2} \frac{\partial W}{\partial \varepsilon_{i k}},
$$

with the Kronecker delta $\delta_{i k}$. With this notation, unnecessary factors of 2 in Eqs. (20) and (23) of our original article are avoided. Consequently, the second and third lines of Eq. (20) should read

$$
\begin{aligned}
& \rho \omega^{2} u_{y}=c_{44} k^{2} u_{y}-i b_{2} k \sin \theta_{0}\left[2 \sin \phi_{0} \cos \phi_{0} \cos \theta_{0} M_{1}+\cos 2 \phi_{0} M_{2}\right], \\
& \rho \omega^{2} u_{z}=c_{44} k^{2} u_{z}+i b_{2} k\left[\sin \phi_{0} \cos \theta_{0} M_{2}-\cos 2 \theta_{0} \cos \phi_{0} M_{1}\right],
\end{aligned}
$$

and Eq. (23) should read

$$
\left[\omega^{2}-v_{t}^{2}\left(1-\frac{F b_{2}^{2}}{v_{t}^{2} \mu_{0} \rho}\left[\chi_{11} w_{5}^{2}+\chi_{22} w_{6}^{2}-\left(\chi_{12}+\chi_{21}\right) w_{5} w_{6}\right]\right) k^{2}\right] u_{z}=0 .
$$

In Eq. (A3), the sign before $D_{\mathrm{s}}$ is wrong. Equation (A3) should read

$$
G_{3}=\left.\partial_{m_{3}} G\right|_{m=m_{0}}=-\mu_{0} H_{3}+2 B_{\mathrm{d}} \cos ^{2} \theta_{0}+2 B_{\mathrm{u}} u_{3}^{2}-D_{\mathrm{s}} k^{2} .
$$

Equation (A14) misses a factor of 2 and should read

$$
\mu_{0}\left(\begin{array}{l}
h_{1} \\
h_{2}
\end{array}\right)=\left(\begin{array}{c}
-2 b_{2} \sin \theta_{0} \cos \theta_{0} \varepsilon_{x y} \\
0
\end{array}\right) .
$$

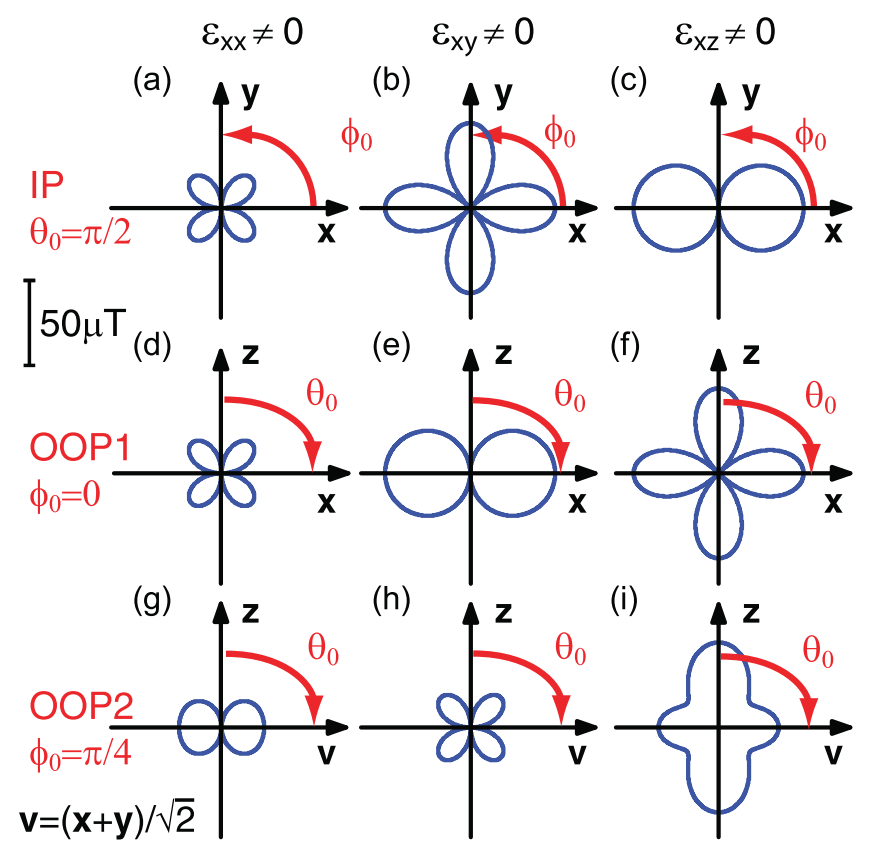

FIG. 1. Corrected Fig. 4. 
The missing factor of 2 in Eq. (A14) resulted in a contour in Fig. 4(h) that is too small by a factor of 2. Figure 1 contains a corrected version of Fig. 4. The figure caption, the paper text, all further figures, and all conclusions remain unaffected.

We thank I. I. Pyataikin for pointing out these errors.

[1] V. V. Lemanov, A. V. Pavlenko, and A. N. Grishmanovskii, Zh. Eksp. Teor. Fiz. 59, 712 (1971) [Sov. Phys. JETP 32, 389 (1971)].

[2] V. V. Lemanov and G. A. Smolenskii, Usp. Fiz. Nauk 108, 465 (1972) [Sov. Phys. Usp. 15, 708 (1973)]. 